Cavendish-HEP-2010/18

October 2010

\title{
Charge asymmetry ratio as a probe of quark flavour couplings of resonant particles at the LHC
}

\author{
C.H. Kom And W.J. StiRling \\ Cavendish Laboratory, University of Cambridge, CB3 0HE, UK
}

\begin{abstract}
We show how a precise knowledge of parton distribution functions, in particular those of the $u$ and $d$ quarks, can be used to constrain a certain class of New Physics models in which new heavy charged resonances couple to quarks and leptons. We illustrate the method by considering a left-right symmetric model with a $W^{\prime}$ from a $S U(2)_{R}$ gauge sector produced in quark-antiquark annihilation and decaying into a charged lepton and a heavy Majorana neutrino. We discuss a number of quark and lepton mixing scenarios, and simulate both signals and backgrounds in order to determine the size of the expected charge asymmetry. We show that various quark $-W^{\prime}$ mixing scenarios can indeed be constrained by charge asymmetry measurements at the LHC, particularly at $\sqrt{s}=14 \mathrm{TeV}$.
\end{abstract}

\section{Introduction}

Many theories beyond the Standard Model (SM) predict existence of new particles that can be discovered at the LHC. Once they are discovered and basic properties such as masses measured, a next important step would be to determine the couplings of these new particles. Determination of these couplings will be crucial in unravelling the structure of the underlying physics models. While this is a non-trivial task, strategies to measure various important model-specific couplings have been proposed.

In this paper, we discuss how precise knowledge of parton distribution functions (PDFs) could provide important information on a particular class of couplings. These are the couplings between quarks and charged resonances that might be produced at the LHC, i.e. via $q \bar{q}^{\prime} \rightarrow X^{ \pm}$.

The fact that the LHC is a proton-proton collider means that for many processes, positively charged (leptonic) final states will be more copiously produced than their negatively charged counterparts. Depending on the typical scales involved, the charge asymmetry ratio

$$
R_{ \pm} \equiv \frac{N_{+}}{N_{-}}
$$


where $N_{+}\left(N_{-}\right)$are number of events with positive(negative) charge assignment, for a given event topology can be determined if the underlying hard process is known 1 In Ref. [1] the variable $R_{ \pm}$ was suggested as a diagnostic tool for the presence of New Physics (NP) with a SM background dominated by $W+n$ jet processes 2 An important advantage of using $R_{ \pm}$is that theoretical uncertainties tend to cancel in the ratio, leading to relatively robust predictions.

Another feature of $R_{ \pm}$is that for (sub)processes produced at the same scale, contributions from different incoming partons will contribute to the ratio differently, due to the non-universal flavour content inherent in the proton. For example, processes initiated by valence quarks $u$ and $d$ will tend to give $R_{ \pm}>1$, since $u(x)>d(x)$, while those initiated by the sea quarks $c, s$ and $b$ will lead to $R_{ \pm} \sim 1$. A measured value of $R_{ \pm}$can therefore lead to a constraint on the relative contribution from these initial state partons. Moreover, as the ratio is independent of the absolute normalisation of the couplings involved, it provides a genuine test of the structure of the parton coupling to the final state particle(s) 3

These ideas can be applied to NP models where new charged particles are produced at resonance at the LHC. The presence of a resonance implies that, in the absence of SM background, $R_{ \pm}$should track closely the ratio of 'weighted' parton luminosities

$$
\frac{\partial \mathcal{L}_{a b}}{\partial M_{V}^{2}}=\frac{1}{s} \int_{y_{\min }}^{y_{\max }} \mathrm{d} y\left|\tilde{V}_{a b}\right|^{2} f_{a}\left(x_{1}, M_{V}^{2}\right) f_{b}\left(x_{2}, M_{V}^{2}\right),
$$

where $x_{1}=\frac{M_{V}}{\sqrt{s}} e^{y}, x_{2}=\frac{M_{V}}{\sqrt{s}} e^{-y}, M_{V}=\sqrt{x_{1} x_{2} s}$ is the mass of the resonance, $\tilde{V}_{a b}$ is its coupling to partons $a$ and $b$, and $\left[y_{\min }, y_{\max }\right]$ is the rapidity interval in which the resonance is produced. We denote such parton luminosity ratio by $\tilde{R}_{ \pm}$. At higher orders, additional QCD radiation will skew the parton luminosity prediction slightly, as will cuts on the decay products of the resonance. Nevertheless it should still be possible to obtain quantitative estimates of $R_{ \pm}$using appropriate Monte Carlo programmes.

Charged resonances that might be discovered at the LHC include, for example, vector bosons in technicolor or extended gauge theories [5, [6], and charged sleptons in standard supersymmetric models with R-parity violation [7]. In the same spirit, SM Higgs-stralung off a (off-shell) $W$ boson is another interesting example, since we would expect $\sigma\left(W^{+} H\right)>\sigma\left(W^{-} H\right)$.

For concreteness, we will focus in this study on a left-right (LR) symmetric model [8] with a heavy $W^{\prime}$ from a $S U(2)_{R}$ gauge sector. The primary leptonic decay mode of the $W^{\prime}$ is $W^{\prime} \rightarrow l N_{l}$, where $N_{l}$ is a heavy Majorana neutrino, followed by three-body decay of $N_{l}$ into a charged lepton and 2 jets. The Majorana nature of $N_{l}$ leads to 'same-sign di-lepton (SSDL) plus 2 jets' events. This mode has not so far been searched for in the Tevatron $(p \bar{p})$ experiments (however see the CDF [9] and D0 [10] $W^{\prime}$ searches in other decay channels). The discovery potential of this model at the $14 \mathrm{TeV}$ LHC has been studied in [11, 12]. We will see that the SM background can be strongly suppressed by applying cuts similar to those in the above studies, together with

\footnotetext{
${ }^{1}$ The paradigm process is of course Standard Model $W^{ \pm}$production, for which $R_{ \pm} \sim 1.33(1.42)$ at $14(7) \mathrm{TeV}$ LHC in NLO QCD perturbation theory [1, 2]. Here the process is initiated primarily by $u \bar{d}$ and $d \bar{u}$ scattering.

${ }^{2}$ For state-of-the-art calculations of $W+n$ jet cross sections in the SM, the reader is referred to Refs. [3, 4].

${ }^{3}$ Of course the statistics and hence the accuracy in determing $R_{ \pm}$will still be affected by the normalisation of the couplings involved.
} 
the requirement that the di-lepton pair has the same charge. As a result, the experimentally determined value of $R_{ \pm}$will be correlated with the parton luminosity predictions. Note that the charge asymmetry ratio is only weakly dependent on the decay modes of the charged resonance. Therefore our discussion can be readily extended to other $W^{\prime}$ models, for example the extended gauge theory model considered in [5, 6] (the discovery potential of this model at the LHC is discussed in [11]), in addition to the models mentioned above.

There exist models with additional constraining flavour structures, for example discrete leftright symmetries relating the $S U(2)_{L}$ and $S U(2)_{R}$ sectors. Without a concrete high-scale model implementation, the flavour/generation dependent couplings may be considered arbitrary [13]. Phenomenologically, if $W^{\prime}$ is relatively light, the quark mixing structure can be strongly constrained by low energy observables such as flavour changing neutral currents (FCNC), for example from $K^{0}-\bar{K}^{0}$ mixing [14]. We will illustrate to what extent different flavour structures can be distinguished using $R_{ \pm}$.

The paper is organised as follows. In the next section we specify our $W^{\prime}$ model and discuss the behaviour of the parton luminosity ratios $\tilde{R}_{ \pm}$given a set of representative quark flavour mixing matrices. In Section 3 we then perform a numerical study to show the relation between $\tilde{R}_{ \pm}$and $R_{ \pm}$, followed by a discussion on the extent to which $R_{ \pm}$can be used to distinguish quark flavour structures at $14 \mathrm{TeV}$ LHC. Finally, we briefly comment on the prospect of using this observable in the early stages of LHC running.

\section{The $W^{\prime}$ model}

The Lagrangian describing the coupling of the $W^{\prime}$ to fermions (quarks and leptons) is given by

$$
\mathcal{L}_{W^{\prime}}=\frac{\tilde{g}}{\sqrt{2}} \tilde{V}_{i j} \bar{u}_{i} \gamma^{\mu} P_{R} d_{j} W_{\nu}^{\prime}+\frac{\tilde{g}}{\sqrt{2}} \tilde{U}_{i j} \bar{e}_{i} \gamma^{\mu} P_{R} N_{j} W_{\nu}^{\prime-}+\text { h.c. }
$$

In the above, we have assumed that the $W^{\prime}$ couples only to right-handed fermions with an overall coupling strength normalised to $\tilde{g}$. The quark and lepton mixings are given by $\tilde{V}$ and $\tilde{U}$ respectively. As our focus is on the effect of the structure of $\tilde{V}$ on $R_{ \pm}$, we will assume, for simplicity, that $\tilde{g}$ is the same as the SM electroweak coupling $g$ and that $\tilde{U}$ is diagonal, i.e. that the two charged leptons from $W^{\prime}$ decay have the same flavour. This allows us to focus on samesign, same-flavour leptonic final states. However for more general $\tilde{U}$, same-sign, different-flavour final states can also be considered.

In some (LR) symmetric models, $\tilde{V}$ is related to the SM CKM matrix, $V_{\mathrm{CKM}}$. In the limit of (pseudo-)manifest LR symmetry, $\tilde{V}=V_{\mathrm{CKM}}$ and the mass of $W^{\prime}$ is constrained to be above a few $\mathrm{TeV}$ [16], primarily by $\Delta F=2$ processes such as $K^{0}-\bar{K}^{0}$ mixing. However this lower bound can be relaxed by considering more general $\tilde{V}$ structures, see for example Ref. [17]. A

\footnotetext{
${ }^{4} \mathrm{~A}$ general interaction Lagrangian is given in [15].
} 


\begin{tabular}{|c|c|}
\hline & $\tilde{V}$ \\
\hline \hline Diagonal mixing & $\tilde{V}_{\mathrm{I}}=\mathbb{I}$ \\
CKM mixing & $\tilde{V}_{\mathrm{CKM}}=V_{\mathrm{CKM}}$ \\
Off-diagonal mixing & $\tilde{V}_{\mathrm{II}}$ (see eq. (44) $)$ \\
1st generation only & $\left(\tilde{V}_{1 \mathrm{st}}\right)_{i j}=\sqrt{3} \delta_{i 1} \delta_{j 1}$ \\
2nd generation only & $\left(\tilde{V}_{2 \mathrm{nd}}\right)_{i j}=\sqrt{3} \delta_{i 2} \delta_{j 2}$ \\
\hline
\end{tabular}

Table 1: Representative quark mixing matrices $\tilde{V}$ in the charged current Lagrangian with a $W^{\prime}$, see Eq. (3). The normalisation of these matrices is such that $\sum_{i j}\left|\tilde{V}_{i j}\right|^{2}=3$.

recent study [18] suggests that if the quark mixing takes the form

$$
|\tilde{V}| \sim\left(\begin{array}{ccc}
0 & \frac{1}{\sqrt{2}} & \frac{1}{\sqrt{2}} \\
1 & 0 & 0 \\
0 & \frac{1}{\sqrt{2}} & \frac{1}{\sqrt{2}}
\end{array}\right),
$$

then right-handed current contributions could lead to sizeable $\mathrm{CP}$ violating effects in $B_{s}$ mixing as suggested by recent results [19], while remaining consistent with stringent $\Delta F=2$ observables for relatively low effective scales. We will take this quark mixing structure, from now on denoted by $\tilde{V}_{\text {II }}$, as one of our 'reference' structures. This can be compared with other representative structures, for example CKM-like mixing $\left(\tilde{V}_{\text {CKM }}\right)$, diagonal mixing $\left(\tilde{V}_{\mathrm{I}}\right)$ and when the $W^{\prime}$ couples

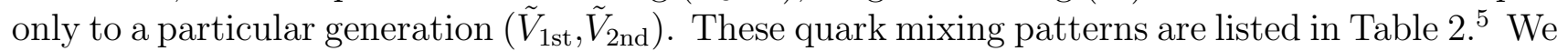
will compare to what extent $R_{ \pm}$can distinguish these different mixing patterns. For simplicity, we assume no mixing between the SM $W$ and the $W^{\prime}$. The decay mode $W^{\prime} \rightarrow W Z$ is forbidden as a result.

As discussed in the Introduction, once $\tilde{V}$ is specified, the ratio of parton luminosities defined in Eq. 2 can be determined. As long as the events selected to determine the charge asymmetry ratio come dominantly from the $W^{\prime}$ signal, $R_{ \pm}$should track the corresponding $\tilde{R}_{ \pm}$closely. We again note that this correspondence should depend only weakly on the event topologies and acceptance cuts.

In Fig. 1 we display $\tilde{R}_{ \pm}$for the various quark mixings shown in Table 2 as a function of $M_{W^{\prime}}$. In these plots, MSTW08 NLO PDF sets [2] are used, with 68\%cl PDF uncertainties also included. Once NLO pQCD corrections are accounted for, the PDF uncertainties are expected to be the most important theoretical uncertainty on the ratio predictions. The factorisation scale is chosen to be equal to the mass of the resonance. As we can see,the ratios for $\tilde{V}_{1 s t}, \tilde{V}_{\mathrm{I}}$ and $\tilde{V}_{\text {CKM }}$ are all quite similar, as they are dominated by contributions from the first generation quarks. However $\tilde{V}_{\text {II }}$ shows a clear deviation from $\tilde{V}_{\mathrm{I}}$ and also $\tilde{V}_{2 \text { nd }}$ when compared with the PDF errors: 6

We can also understand the general behaviour of $\tilde{R}_{ \pm}$for different quark mixing patterns. As $M_{W^{\prime}}$ increases, successively higher parton momentum fractions $x$ are probed. While the

\footnotetext{
${ }^{5}$ The matrices are normalised according to $\sum_{i j}\left|\tilde{V}_{i j}\right|^{2}=3$.

${ }^{6}$ Note that early LHC measurements of the charge asymmetry in SM $W^{ \pm}$production will very likely reduce the PDF errors further.
} 
$u / d$ ratio increases monotonically with $x$, the $\bar{d} / \bar{u}$ ratio first increases and then decreases as $x$ increases in the relevant range. The fractional decrease of the latter overcomes the increase of the $u / d$ ratio, leading to an overall slight decrease of the parton luminosity ratio for $\tilde{V}_{1 s t}, \tilde{V}_{\mathrm{I}}$ and $\tilde{V}_{\mathrm{CKM}}$ in the region $M_{W^{\prime}}=3-4 \mathrm{TeV}$. On the other hand, the luminosity ratios for the second and third generation partons are much flatter functions of $x$, leading to an increasing $\tilde{R}_{ \pm}$ primarily controlled by the $u / d$ ratio for $\tilde{V}_{\mathrm{II}}$, and a $\tilde{R}_{ \pm}$ratio close to 1 for $\tilde{V}_{2 \text { nd }}$.
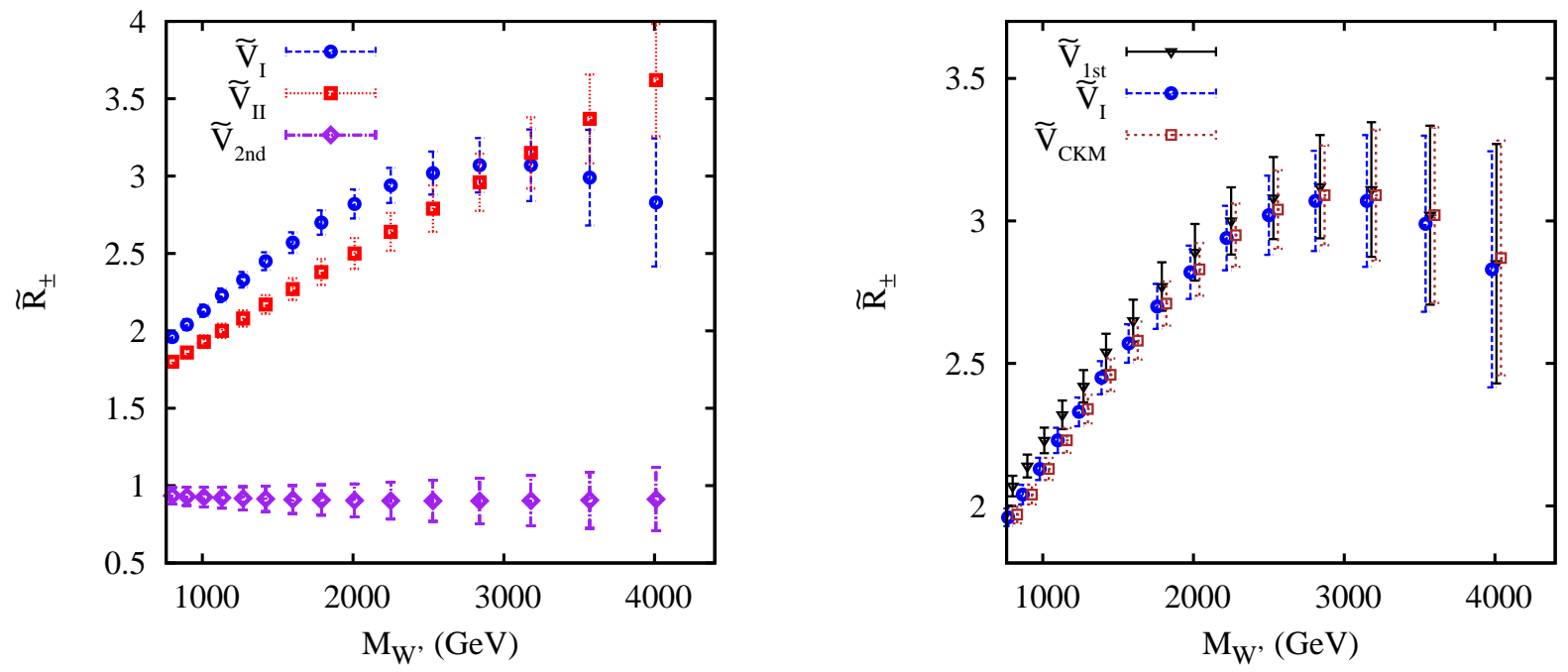

Figure 1: Variation of parton luminosity ratios $\tilde{R}_{ \pm}$with $M_{W^{\prime}}$ at $14 \mathrm{TeV}$ LHC. The quark mixing matrices $\tilde{V}$ are defined in Table 2. The rapidity $y$ of $W^{\prime}$ satisfies $|y|<2.5$. The ratio is computed using the MSTW08 NLO PDF set [2] with factorisation scale $\mu_{F}=M_{W^{\prime}}$. The error bars correspond to $68 \% \mathrm{cl}$ PDF errors.

In the next section, we will perform event simulations to quantify the correlations between $\tilde{R}_{ \pm}$and $R_{ \pm}$. We comment at this point that both values depend on the PDF sets used. For example, our simulation is performed using Herwig++2.4.2 [20], interfaced with the LHAPDF library [21], with MRST LO** as the default PDF set. Compared to MRST LO**, we find significant differences for the predicted values of $\tilde{R}_{ \pm}$and hence $R_{ \pm}$when using MSTW08 NLO PDFs (interfaced via the LHAPDF library [21]). This can be understood by considering the differences in individual PDFS between the recent MSTW08 and earlier MRST LO** sets. Of course a detailed simulation of $W^{\prime}$ production would require a proper NLO calculation using an up-to-date NLO PDF set such as MSTW08. We expect that the bulk of the NLO corrections comes from the differences between the LO and NLO PDF sets (see, for example, Ref. [1]), and so for this reason we will use MSTW08 NLO as our preferred PDF choice. Using other PDF sets, for example CTEQ6.6(NLO) [22], will also lead to slightly different central values and PDF uncertainties. The discrepancies between different PDF sets will hopefully be resolved in the future. 


\section{Numerical study}

In this section we discuss the basic properties of the signal and backgrounds calculated using Herwig++2.4.2. A set of cuts is then proposed to extract the signal from the background, before the prospect of quantitatively measuring $R_{ \pm}$is discussed.

As discussed in the Introduction, the signal is same-sign di-leptons in association with 2 hadronic jets. We assume that the heavy Majorana neutrinos are lighter than the $W^{\prime}$. Clearly, an accurate measurement of $R_{ \pm}$will require much higher statistics than a $W^{\prime}$ discovery. We will therefore investigate a low mass range $M_{W^{\prime}}=1-2 \mathrm{TeV}$. For simplicity, the masses of the 3 heavy Majorana neutrinos are assumed to be degenerate, and are given by $M_{N}=\frac{1}{2} M_{W^{\prime}}$.

To identify a signal event, the basic strategy is to look for a high $p_{T}$ SSDL pair and a pair of high $p_{T}$ jets coming from $W^{\prime} \rightarrow l N_{l} \rightarrow l l j j$. The invariant mass of these four objects will lead to a resonance peak for the $W^{\prime}$. One of the two lepton-jet-jet combinations will also give a resonance peak for $N_{l}$.

The dominant background is expected to come from $t \bar{t}$. Compared with the studies in $[11,12]$, the requirement of SSDL efficiently suppresses this background. This is because at the hadron level, one of the leptons must come from $B$ decay (the leptons from the two $W$ decays naturally have opposite sign), which in general does not satisfy a lepton isolation requirement. Other potential backgrounds are from $W b \bar{b} j$ and $W Z\left(\gamma^{*}\right) j j$ production. The former process will again be suppressed by a lepton isolation requirement. However its production cross section is relatively large, and therefore it demands more attention. The latter $W Z\left(\gamma^{*}\right) j j$ process can lead to SSDL +2 jet events if the wrong-sign lepton from $Z\left(\gamma^{*}\right) \rightarrow l^{+} l^{-}$is not identified, for example when it falls outside the central tracking region.

Note that since the $t \bar{t}$ background is produced predominantly by $g g$ scattering, we have $R_{ \pm} \sim 1$ for this process, i.e. any final state involving charged leptons is symmetric under change of sign, whereas $R_{ \pm}$from $W b \bar{b} j$ and $W Z\left(\gamma^{*}\right) j j$ is generally $>1$ since these arise from $q \bar{q}^{\prime}$ and $q g$ scatterings.

In terms of other possible backgrounds, we expect that by requiring SSDL, the $Z\left(\gamma^{*}\right)$ background discussed in [11, 12] will be suppressed to a negligible level. The $W^{ \pm} W^{ \pm} j j$ background will also be insignificant due to its small production rate [23, 24]. Therefore these processes will not be discussed any further. We also do not attempt to study detector effects. For this reason, the QCD multijet background is not considered. A discussion of this background and its relevance for $W^{\prime}$ discovery, including a detailed detector simulation, can be found in Ref. [11]. Note that any contamination of the $W^{\prime} \rightarrow l l j j$ signal from QCD multijet production will tend to reduce $R_{ \pm}$since this background is expected to be charge symmetric.

To simulate the $W^{\prime}$ signal, we implement the $W^{\prime}$ model into Herwig++. The latter is also used to generate both the signal and the $t \bar{t}$ background. We use MSTW08 NLO PDFs together with default values for other parameters. The $W Z\left(\gamma^{*}\right) j j$ and $W b \bar{b} j$ processes are simulated using ALPGEN [25], with Herwig6.510 [26] then generating the parton showers. Parton level cuts appropriate to the experimental cuts discussed below are imposed to speed up the event generation, and we use 'out of the box' SM parameters for these processes.

An event is selected for further analysis based on the following criteria: 
- the leptons (jets) should lie within pseudorapitidy range $|\eta|<2.5$ (4.5);

- a lepton is isolated if $E_{\mathrm{ISO}}^{l} \leq E_{\mathrm{ISO}}^{\mathrm{min}}=5 \mathrm{GeV}$, where $E_{\mathrm{ISO}}^{l}$ is the transverse energy in a cone of $R=0.4$ surrounding the lepton;

- the 2 hightest $p_{T}$ leptons must have the same sign and flavour;

- the 2 highest $p_{T}$ jets and the 2 highest $p_{T}$ leptons must be separated by $\Delta R \geq 0.4$.

In the above, an isolated lepton is assumed to be identified with $100 \%$ efficiency when $p_{T}^{l}>$ $10 \mathrm{GeV}$. Muons are assumed to be invisible when $\left|\eta_{\mu}\right|>2.5$. Our jet reconstructions are performed using FastJet-2.4.2 [27] using the anti- $k_{T}$ algorithm [28] with $R=0.4$.

We then apply further cuts to improve the signal/background ratio:

1. select events with $\geq 2$ isolated leptons with $p_{T}^{l}>75 \mathrm{GeV}$ and $\geq 2$ jets with $p_{T}^{j}>50 \mathrm{GeV}$;

2. veto an event if a third lepton with $p_{T}^{l}>10 \mathrm{GeV}$ is present which has the same flavour but opposite charge to the two hardest $p_{T}^{l}$ leptons;

3. retain an event when the invariant mass of the 2 leptons and 2 jets, $m_{l l j j}$, satisfies

$$
0.7 M_{W^{\prime}}^{\mathrm{rec}}<m_{l l j j}<1.2 M_{W^{\prime}}^{\mathrm{rec}}
$$

Here $M_{W^{\prime}}^{\text {rec }}$ is the value of the reconstructed $W^{\prime}$ mass.

Of course the backgrounds considered will have missing transverse energy $E_{T}$ already at the parton level. Furthermore leptons from $B$ decays can have displaced vertices. These can be used as additional handles to further suppress the backgrounds. However we refrain from doing so here in an attempt to remain conservative. The leading-order multiparticle final-state backgrounds are known to suffer from scale uncertainties, and so the values quoted below should be treated as rough estimates only.

Due to tight lepton isolation and hard lepton $p_{T}$ cut, we find that the $W b \bar{b} j$ background is suppressed to negligible level. We provide a numerically more conservative estimate using MCFM [3] (at leading order). This parton level simulation does not model semi-leptonic decay of the $b \mathrm{~s}$, rather a 'rule of thumb' efficiency factor $\epsilon_{B} \sim 1 / 200$ is used for the probability of obtaining an isolated lepton of a given flavour (i.e. $e$ or $\mu$ ) from $B$ decay suggested recently in [29].

In Table 2 we display the effect of the selection cuts for $M_{W^{\prime}}=1.0,1.5,2.0 \mathrm{TeV}$ with the quark mixing structures $\tilde{V}_{\mathrm{I}}$ and $\tilde{V}_{\mathrm{II}}$. $\sigma_{1,2,3}$ refer to the cross sections after applying the cuts 1 (hard leptons and jets), 2 (opposite-sign, same-flavour veto (OSSFV)) and 3 (lljj invariant mass constraint) defined above, including the ++ and -- contributions from both electrons and muons. In general, the signal cross sections for $\tilde{V}_{\text {II }}$ are smaller than those for $\tilde{V}_{\mathrm{I}}$, reflecting the different PDF combinations entering the processes via off-diagonal and diagonal flavour couplings respectively. Applying the $M_{W^{\prime}}$ invariant mass cut further suppresses the backgrounds by a factor of a few, while retaining most of the signal as expected. Our more conservative estimate of the $W b \bar{b} j$ background is still relatively small compared with the $t \bar{t}$ background. For the 


\begin{tabular}{|c|c|cccc|}
\hline$M_{W^{\prime}}$ & Process & $\sigma_{\text {tot }}$ & $\sigma_{1}$ & $\sigma_{2}$ & $\sigma_{3}$ \\
\hline \hline \multirow{5}{*}{$1.0 \mathrm{TeV}$} & $W^{\prime}\left(\tilde{V}_{\mathrm{I}}\right)$ & $4.78 \cdot 10^{3}$ & $1.19 \cdot 10^{3}$ & $1.15 \cdot 10^{3}$ & $1.02 \cdot 10^{3}$ \\
& $W^{\prime}\left(\tilde{V}_{\mathrm{II}}\right)$ & $2.62 \cdot 10^{3}$ & 647 & 622 & 542 \\
\cline { 2 - 6 } & $t \bar{t}$ & $6.06 \cdot 10^{5}$ & 11 & 9.7 & 2.8 \\
& $W Z\left(\gamma^{*}\right) j j$ & - & 3.0 & 0.83 & 0.37 \\
& $W b \bar{b} j$ & - & $115 \epsilon_{B}$ & $115 \epsilon_{B}$ & $80 \epsilon_{B}$ \\
\hline \hline \multirow{5}{*}{$1.5 \mathrm{TeV}$} & $W^{\prime}\left(\tilde{V}_{\mathrm{I}}\right)$ & 882 & 289 & 276 & 253 \\
& $W^{\prime}\left(\tilde{V}_{\mathrm{II}}\right)$ & 411 & 135 & 128 & 116 \\
\cline { 2 - 6 } & $t \bar{t}$ & $6.06 \cdot 10^{5}$ & 11 & 9.7 & 1.4 \\
& $W Z\left(\gamma^{*}\right) j j$ & - & 3.0 & 0.83 & 0.22 \\
& $W b \bar{b} j$ & - & $115 \epsilon_{B}$ & $115 \epsilon_{B}$ & $41 \epsilon_{B}$ \\
\hline \hline \multirow{5}{*}{$2.0 \mathrm{TeV}$} & $W^{\prime}\left(\tilde{V}_{\mathrm{I}}\right)$ & 226 & 81.8 & 77.8 & 72.9 \\
& $W^{\prime}\left(\tilde{V}_{\mathrm{II}}\right)$ & 92.8 & 33.6 & 31.7 & 29.3 \\
\cline { 2 - 6 } & $t \bar{t}$ & $6.06 \cdot 10^{5}$ & 11 & 9.7 & 0.53 \\
& $W Z\left(\gamma^{*}\right) j j$ & - & 3.0 & 0.83 & 0.14 \\
& $W b \bar{b} j$ & - & $115 \epsilon_{B}$ & $115 \epsilon_{B}$ & $19 \epsilon_{B}$ \\
\hline
\end{tabular}

Table 2: Cross sections (in fb) for different signal and background processes at $14 \mathrm{TeV}$ LHC. All 4 same-sign, same-flavour di-lepton channels are included. $\sigma_{1,2,3}$ correspond to the cross sections after applying cuts 1,2,3 described in the text. $\epsilon_{B}$ denotes the efficiency in extracting an isolated lepton from a $b$ quark and is estimated to be $\sim 1 / 200$ [29]. See Table 3 for the simulated $R_{ \pm}$ values.

$W Z\left(\gamma^{*}\right) j j$ background, the OSSFV is shown to be efficient. In the following we will therefore neglect the $W b \bar{b} j$ and $W Z\left(\gamma^{*}\right) j j$ contributions when performing the $R_{ \pm}$estimation.

In Fig. 2 we show some sample kinematic distributions (normalised to $5 \mathrm{fb}^{-1}$ ) from different processes. For clarity, only $W^{\prime}$ signals with $\tilde{V}_{\mathrm{I}}$ mixing and the dominant $t \bar{t}$ background are shown. We see clearly the $W^{\prime}$ and $N_{l}$ resonance peaks from the invariant masses of $l l j j$ and $l j j$ respectively. The $l j j$ peak from the second hardest lepton is more pronounced due to our assumed relation $M_{N}=\frac{1}{2} M_{W^{\prime}}$, which results in higher average $p_{T}$ for the lepton from the decay of the $W^{\prime}$ than that from the decay of the $N_{l}$.

We also see that more pure samples can readily be obtained by varying the cut values, for example the $p_{T}$ of the second hardest lepton, as a function of $M_{W^{\prime}}$. Additional cuts, for example on the invariant mass of $M_{N}$, may also be useful. Given that we already have fairly pure samples we refrain from optimising the cuts further. Other strategies for extracting the signal can be found in Refs. [11, 12].

As discussed earlier, the focus of this work is on the extraction of $R_{ \pm}$. In Table 3 , a comparison between $\tilde{R}_{ \pm}$and the simulated $R_{ \pm}$is shown. The cross-section ratios at the hard process level obtained from Herwig++2.4.2 are well within the PDF uncertainties of the parton luminosity 


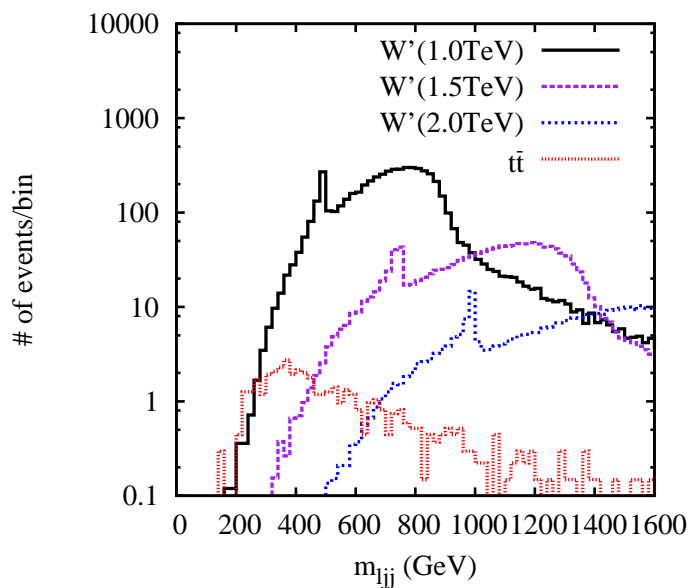

(a) $m_{l_{1} j j}$

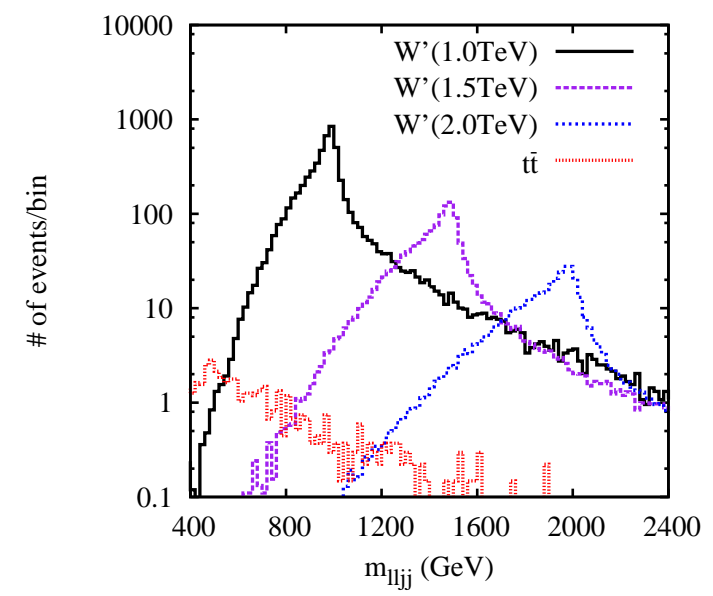

(c) $m_{l l j j}$

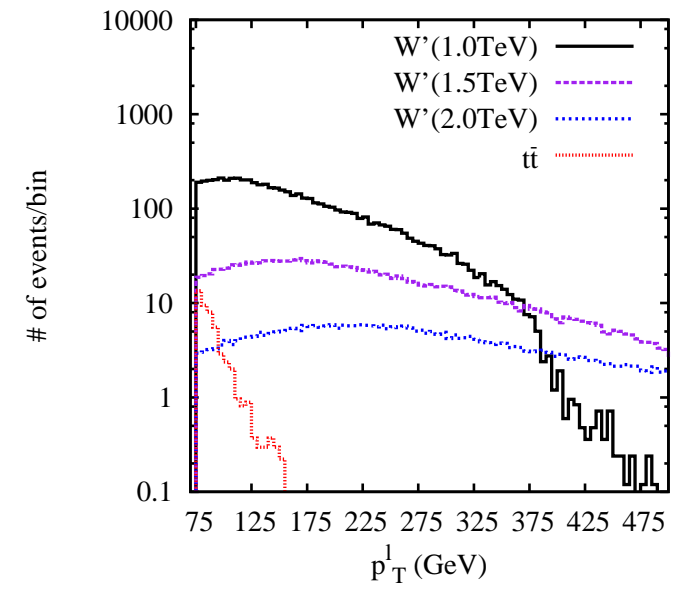

(e) $p_{T}$ (2nd hardest lepton)

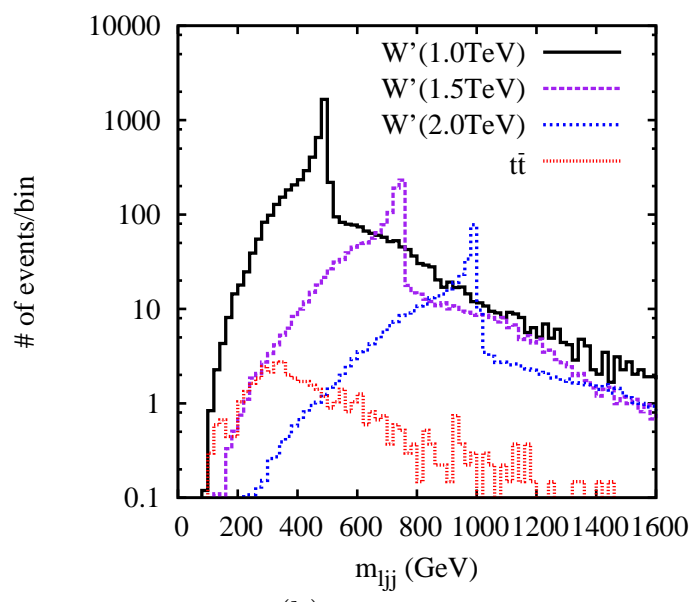

(b) $m_{l_{2} j j}$

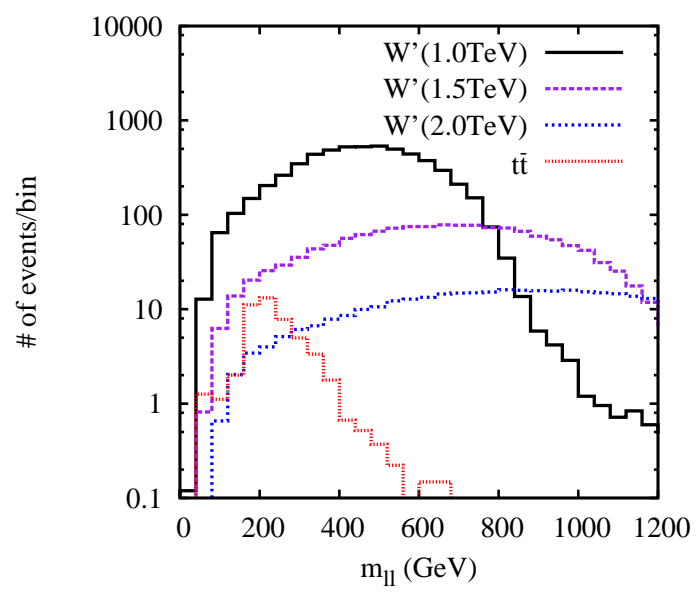

(d) $m_{l l}$

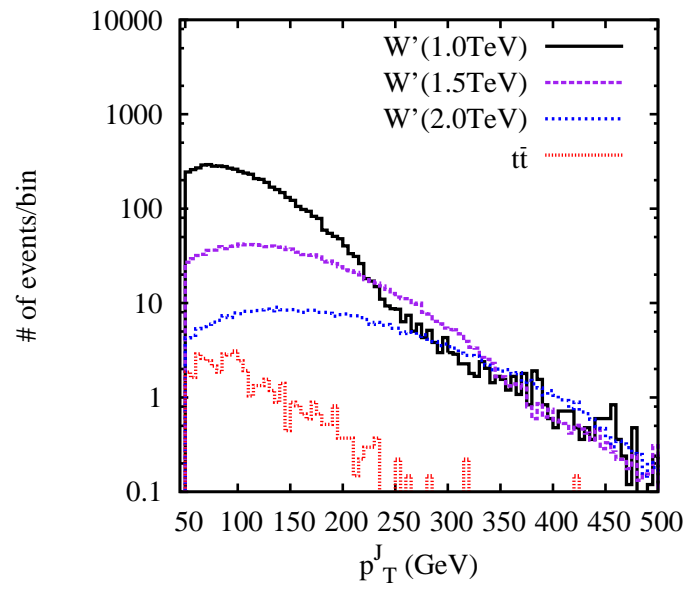

(f) $p_{T}$ (2nd hardest jet)

Figure 2: Selected kinematic distributions for the same-sign dilepton events. The number of events are normalised to $5 \mathrm{fb}^{-1}$. 


\begin{tabular}{|c|cc|cc||cc|}
\hline \multirow{2}{*}{$M_{W^{\prime}}$} & \multicolumn{2}{|c|}{$\tilde{V}_{\mathrm{I}}$} & \multicolumn{2}{|c||}{$\tilde{V}_{\mathrm{II}}$} & $t \bar{t}$ & $W Z\left(\gamma^{*}\right) j j$ \\
\cline { 2 - 7 } & $\tilde{R}_{ \pm}$ & $R_{ \pm}$ & $\tilde{R}_{ \pm}$ & $R_{ \pm}$ & $R_{ \pm}$ & $R_{ \pm}$ \\
\hline \hline $1.0 \mathrm{TeV}$ & $2.12(4)$ & $1.99(1)$ & $1.92(4)$ & $1.79(2)$ & $1.0(1)$ & $1.2(2)$ \\
$1.5 \mathrm{TeV}$ & $2.50(6)$ & $2.42(3)$ & $2.21(7)$ & $2.13(4)$ & $1.0(2)$ & $1.1(3)$ \\
$2.0 \mathrm{TeV}$ & $2.82(9)$ & $2.74(7)$ & $2.49(10)$ & $2.40(10)$ & $1.1(4)$ & $1.2(3)$ \\
\hline
\end{tabular}

Table 3: Comparison between parton luminosity ratio $\tilde{R}_{ \pm}$and observed charged asymmetry ratios $R_{ \pm}$(MSTW NLO) at $14 \mathrm{TeV}$ LHC. For the signal, Poisson uncertainties for the $R_{ \pm}$'s at $100 \mathrm{fb}^{-1}$ and $1 \sigma \mathrm{PDF}$ uncertainties for $\tilde{R}_{ \pm}$are displayed in brackets. For the backgrounds, we show the statistical uncertainties based on the number of events generated. The $W b \bar{b} j$ results are not shown due to too few statistics.

ratio predictions, while the values of $R_{ \pm}$are systematically smaller than that of $\tilde{R}_{ \pm}$. The latter is due to the finite acceptance in lepton pseudorapidity and the fact that the average parton $x$ for an up quark is higher than that of a down quark. The $W^{\prime+}$, and hence the $l^{+}$, will tend to have a broader rapidity distribution than that of the $W^{\prime-}$ and $l^{-}$, leading to a larger acceptance loss of the $l^{+}$. It is expected that while the extracted $R_{ \pm}$values will depend slightly on the cuts applied, they will nevertheless track $\tilde{R}_{ \pm}$closely.

In Fig. 3, we compare $\tilde{R}_{ \pm}$and $R_{ \pm}$as a function of $M_{W^{\prime}}$, with quark mixings $\tilde{V}_{\mathrm{I}}$ and $\tilde{V}_{\mathrm{II}}$ as examples, at $14 \mathrm{TeV}$ LHC with an assumed luminosity of $30 \mathrm{fb}^{-1}$. For simplicity, we have not included the background contributions to $R_{ \pm}$. From Table 3 we see that the latter have $R_{ \pm}$ values much closer to 1 . Together with results in Table 2, including background contributions would lower the $R_{ \pm}$values slightly by $\mathcal{O}(1-2) \%$. We see that for $M_{W^{\prime}}$ close to $1 \mathrm{TeV}$, the two mixing structures are clearly distinguishable. At this luminosity, the uncertainties on $R_{ \pm}$ may be expected to come primarily from the PDFs. Statistical uncertainties become comparable at $M_{W^{\prime}} \sim 1.5 \mathrm{TeV}$. However at this luminosity, the ability to distinguish $\tilde{V}_{\mathrm{I}}$ from $\tilde{V}_{\text {II }}$ becomes limited when $M_{W^{\prime}}$ approaches $2 \mathrm{TeV}$.

Finally, given the fact that in the $M_{W^{\prime}}=\mathcal{O}(1.0) \mathrm{TeV}$ region the $W^{\prime}$ can be easily detected, we discuss the extent to which early LHC data (i.e. $\sim 1 \mathrm{fb}^{-1}$ at $7 \mathrm{TeV}$ ) can measure $R_{ \pm}$. Measuring $R_{ \pm}$at different centre of mass energies will probe different parton $x$ values, and hence could provide additional information to discriminate different PDF choices.

In Table 4, we show the results for the signal processes at $7 \mathrm{TeV}$, assuming an integrated luminosity of $1 \mathrm{fb}^{-1}$. Using the same cuts as the $14 \mathrm{TeV}$ case, the backgrounds we have considered are again shown to be tiny $\left(\sigma(t \bar{t})<1 \mathrm{fb}, \sigma\left(W Z\left(\gamma^{*}\right) j j\right)<0.2 \mathrm{fb}\right.$ and $\sigma(W b \bar{b} j)$ negligible after cut 2 ). We see that while $W^{\prime}$ could be discovered easily, the assumed total luminosity is only able to provide marginal discriminating power between $\tilde{V}_{\mathrm{I}}$ and $\tilde{V}_{\mathrm{II}}$ for $M_{W^{\prime}}$ below $1 \mathrm{TeV}$. However such low values of $M_{W^{\prime}}$ may have difficulty evading the bounds from EW precision observables already discussed. 


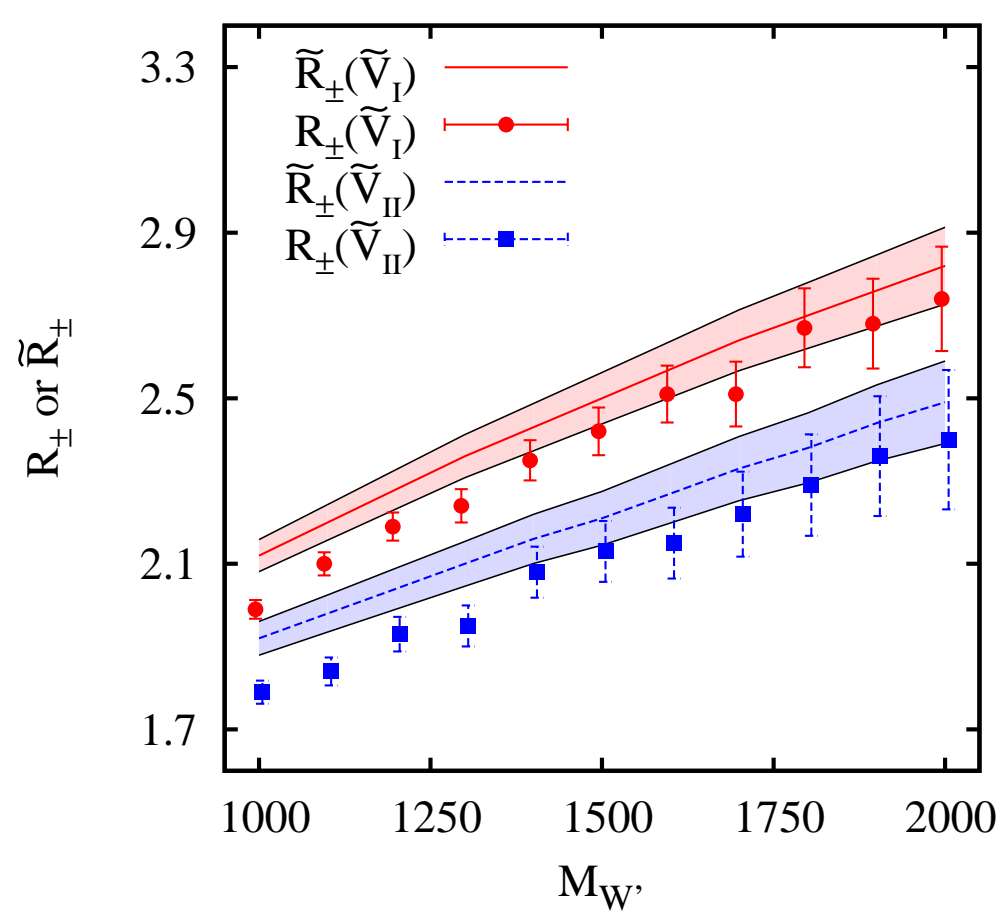

Figure 3: Comparison between parton luminosity ratio $\tilde{R}_{ \pm}$and observed charged asymmetry ratios $R_{ \pm}$(MSTW NLO) at $14 \mathrm{TeV}$ LHC as a function of $M_{W^{\prime}}$. Results from two quark mixing patterns, $\tilde{V}_{\mathrm{I}}$ (upper, red curves) and $\tilde{V}_{\text {II }}$ (lower, green curves) are shown. The colour bands correspond to $1 \sigma \mathrm{PDF}$ uncertainties, while the error bars are Poisson uncertainties on $R_{ \pm}$at 30 $\mathrm{fb}^{-1}$.

\section{Conclusions}

In this paper we have demonstrated how by measuring the charge asymmetry ratio $R_{ \pm}$at the LHC, non-trivial information on the couplings of new heavy charged resonances to quarks may be obtained. We have focussed in particular on the $W^{\prime}$ model, confirming expectations in the literature that in the $M_{W^{\prime}} \sim \mathcal{O}(\mathrm{TeV})$ region the physics backgrounds can be highly suppressed by applying appropriate cuts in the same-sign di-lepton +2 jet channel. As a result, the measured $R_{ \pm}$value and the parton luminosity ratio $\tilde{R}_{ \pm}$are related in a directly correlated manner. Since the value of $\tilde{R}_{ \pm}$can be calculated precisely given a particular flavour mixing structure, we have established a method to constrain the quark mixing given a measured value of $R_{ \pm}$. Although we have considered overall event rates only, with higher luminosity it should be possible to study the rapidity dependence of the charge asymmetry ratio and this would provide additional constraints.

Quantitatively, we have shown that at $14 \mathrm{TeV}$ LHC three classes of representative flavour structures, namely $\tilde{V}_{(\mathrm{I}, \mathrm{CKM}, 1 \mathrm{st})}, \tilde{V}_{\mathrm{II}}$ and $\tilde{V}_{2 \text { nd }}$, can be clearly distinguished with realistic luminosi- 


\begin{tabular}{|c|c|cc|cc|}
\hline$M_{W^{\prime}}$ & Process & $\sigma_{\text {tot }}$ & $\sigma_{3}$ & $\tilde{R}_{ \pm}$ & $R_{ \pm}$ \\
\hline \hline \multirow{2}{*}{$0.8 \mathrm{TeV}$} & $W^{\prime}\left(\tilde{V}_{\mathrm{I}}\right)$ & $2.88 \cdot 10^{3}$ & $5.1 \cdot 10^{2}$ & $2.57(7)$ & $2.4(2)$ \\
& $W^{\prime}\left(\tilde{V}_{\mathrm{II}}\right)$ & $1.26 \cdot 10^{3}$ & $2.2 \cdot 10^{2}$ & $2.26(7)$ & $2.2(3)$ \\
\hline \hline \multirow{2}{*}{$1.0 \mathrm{TeV}$} & $W^{\prime}\left(\tilde{V}_{\mathrm{I}}\right)$ & $9.98 \cdot 10^{2}$ & $2.4 \cdot 10^{2}$ & $2.81(9)$ & $2.8(4)$ \\
& $W^{\prime}\left(\tilde{V}_{\mathrm{II}}\right)$ & $4.0 \cdot 10^{2}$ & 92 & $2.49(10)$ & $2.4(5)$ \\
\hline \hline \multirow{2}{*}{$1.2 \mathrm{TeV}$} & $W^{\prime}\left(\tilde{V}_{\mathrm{I}}\right)$ & 381 & 105 & $2.99(12)$ & $2.9(6)$ \\
& $W^{\prime}\left(\tilde{V}_{\mathrm{II}}\right)$ & 142 & 38 & $2.71(14)$ & $2.6(10)$ \\
\hline
\end{tabular}

Table 4: Cross sections (in fb), $\tilde{R}_{ \pm}$and $R_{ \pm}$for different $W^{\prime}$ models at $7 \mathrm{TeV}$ LHC. All 4 samesign, same-flavour di-lepton channels are included. $\sigma_{3}$ corresponds to the cross section after applying cut 3 described in the text. The brackets for $\tilde{R}_{ \pm}$are $\pm 1 \sigma$ PDF uncertainties, whereas those for $R_{ \pm}$are Poisson uncertainties at $1 \mathrm{fb}^{-1}$ luminosity. After applying cut 2 , the background processes are suppressed below $1 \mathrm{fb}$, and hence their values are not shown.

ties. However the prospect at $7 \mathrm{TeV}$ LHC is less optimistic due to the lower overall luminosity as well as the larger PDF uncertainties in the higher parton $x$ region being probed.

A knowledge of quark flavour mixing structure will clearly have important implications on EW precision observables. Interestingly, if $W^{\prime}$ is relevant for neutrinoless double beta decay $(0 \nu \beta \beta)$, then measurement of $R_{ \pm}{ }^{7}$ will be crucial in estimating its contribution to $0 \nu \beta \beta$, as only the first generation quarks participate in this low energy process. The same consideration will apply in relating an observation of resonant selectron production in R-parity violating SUSY models to its contribution to $0 \nu \beta \beta$ [30].

Finally, the measurement of $R_{ \pm}$can be extended to signals other than resonance production. We will study the application of this observable in other NP models, for example SUSY, in forthcoming publications.

\section{Acknowledgements}

This work has been partially supported by the Isaac Newton Trust at the University of Cambridge. We thank the members of the Cambridge SUSY working group for useful conversations. CHK thanks Andreas Papaefstathiou for invaluable help in using Herwig++.

\section{References}

[1] C. H. Kom and W. J. Stirling, Eur. Phys. J. C 69 (2010) 67 [arXiv:1004.3404 [hep-ph]].

[2] A. D. Martin, W. J. Stirling, R. S. Thorne and G. Watt, Eur. Phys. J. C 63 (2009) 189 [arXiv:0901.0002 [hep-ph]].

\footnotetext{
${ }^{7}$ In this case, one should focus on the di-electron channel.
} 
[3] J. M. Campbell and R. K. Ellis, Phys. Rev. D 65 (2002) 113007 [arXiv:hep-ph/0202176];

J. M. Campbell and R. K. Ellis, http://mcfm.fnal.gov/.

[4] C. F. Berger et al., Phys. Rev. Lett. 102 (2009) 222001 [arXiv:0902.2760 [hep-ph]];

C. F. Berger et al., Phys. Rev. D 80 (2009) 074036 [arXiv:0907.1984 [hep-ph]].

C. F. Berger et al., arXiv:1009.2338 [hep-ph].

[5] G. Altarelli, B. Mele and M. Ruiz-Altaba, Z. Phys. C 45 (1989) 109 [Erratum-ibid. C 47, $676(1990)]$.

[6] P. Ramond, Ann. Rev. Nucl. Part. Sci. 33 (1983) 31.

[7] H. K. Dreiner, P. Richardson and M. H. Seymour, Phys. Rev. D 63, 055008 (2001) [arXiv:hep-ph/0007228].

[8] J. C. Pati and A. Salam, Phys. Rev. D 10, 275 (1974) [Erratum-ibid. D 11, 703 (1975)].

R. N. Mohapatra and J. C. Pati, Phys. Rev. D 11, 566 (1975).

R. N. Mohapatra and J. C. Pati, Phys. Rev. D 11, 2558 (1975).

G. Senjanovic and R. N. Mohapatra, Phys. Rev. D 12, 1502 (1975).

G. Senjanovic, Nucl. Phys. B 153, 334 (1979).

[9] T. Aaltonen et al. [The CDF Collaboration], Phys. Rev. Lett. 104 (2010) 241801 [arXiv:1004.4946 [hep-ex]].

T. Aaltonen et al. [CDF Collaboration], Phys. Rev. Lett. 103 (2009) 041801 [arXiv:0902.3276 [hep-ex]].

A. Abulencia et al. [CDF Collaboration], Phys. Rev. D 75 (2007) 091101 [arXiv:hepex/0611022].

[10] V. M. Abazov et al. [D0 Collaboration], Phys. Rev. Lett. 100 (2008) 031804 [arXiv:0710.2966 [hep-ex]].

V. M. Abazov et al. [D0 Collaboration], Phys. Rev. Lett. 100 (2008) 211803 [arXiv:0803.3256 [hep-ex]].

[11] G. Aad et al. [The ATLAS Collaboration], arXiv:0901.0512 [hep-ex].

[12] A. Ferrari et al., Phys. Rev. D 62 (2000) 013001.

[13] C. Amsler et al. [Particle Data Group], Phys. Lett. B 667 (2008) 1.

[14] G. Beall, M. Bander and A. Soni, Phys. Rev. Lett. 48 (1982) 848.

[15] A. Papaefstathiou and O. Latunde-Dada, JHEP 0907 (2009) 044 [arXiv:0901.3685 [hep$\mathrm{ph}]]$.

[16] A. Maiezza, M. Nemevsek, F. Nesti and G. Senjanovic, arXiv:1005.5160 [hep-ph].

[17] P. Langacker and S. Uma Sankar, Phys. Rev. D 40 (1989) 1569. 
[18] A. J. Buras, K. Gemmler and G. Isidori, arXiv:1007.1993 [hep-ph].

[19] T. Aaltonen et al. [CDF Collaboration], Phys. Rev. Lett. 100 (2008) 161802 [arXiv:0712.2397 [hep-ex]].

V. M. Abazov et al. [D0 Collaboration], Phys. Rev. Lett. 101 (2008) 241801 [arXiv:0802.2255 [hep-ex]].

V. M. Abazov et al. [D0 Collaboration], Phys. Rev. D 82 (2010) 032001 [arXiv:1005.2757 [hep-ex]].

[20] M. Bahr et al., Eur. Phys. J. C 58 (2008) 639 [arXiv:0803.0883 [hep-ph]].

[21] M. R. Whalley, D. Bourilkov and R. C. Group, arXiv:hep-ph/0508110.

[22] P. M. Nadolsky et al., Phys. Rev. D 78 (2008) 013004 [arXiv:0802.0007 [hep-ph]].

[23] J. R. Gaunt, C. H. Kom, A. Kulesza and W. J. Stirling, Eur. Phys. J. C 69 (2010) 53 [arXiv:1003.3953 [hep-ph]].

[24] T. Melia, K. Melnikov, R. Rontsch and G. Zanderighi, arXiv:1007.5313 [hep-ph].

[25] M. L. Mangano, M. Moretti, F. Piccinini, R. Pittau and A. D. Polosa, JHEP 0307 (2003) 001 [arXiv:hep-ph/0206293].

[26] G. Corcella et al., arXiv:hep-ph/0210213.

[27] M. Cacciari and G. P. Salam Phys. Lett. B 641 (2006) 57. [arXiv:hep-ph/0512210] http://fastjet.fr/

[28] M. Cacciari, G. P. Salam and G. Soyez, JHEP 0804 (2008) 063 [arXiv:0802.1189 [hep-ph]].

[29] Z. Sullivan and E. L. Berger, Phys. Rev. D 82 (2010) 014001 [arXiv:1003.4997 [hep-ph]].

[30] B. C. Allanach, C. H. Kom and H. Päs, Phys. Rev. Lett. 103 (2009) 091801 [arXiv:0902.4697 [hep-ph]].

B. C. Allanach, C. H. Kom and H. Päs, JHEP 0910 (2009) 026 [arXiv:0903.0347 [hep-ph]]. 